\title{
Sequential microbiological monitoring of tracheal aspirates in intubated patients admitted to a pediatric intensive care unit
}

\author{
Mitsuru Miyaki *
}

\begin{abstract}
Ventilator-associated pneumonia (VAP) is an infection that develops within 48 hours or more after the implementation of mechanical ventilation (MV) and, depending on the place where the study is conducted and on the age of the patient, it may be the first or second most frequent cause of nosocomial infection in the PICU. ${ }^{1-3}$ Its incidence varies according to the diagnostic criteria and to the place where the study is performed, ranging from 1.8 to $28.3 \%$ among pediatric patients submitted to MV.1,4-6
\end{abstract}

The gold standard for the diagnosis of VAP is the autopsy (or pathoanatomical examination), as, unfortunately, there is no methodology that can be routinely used in clinical practice with appropriate sensitivity and specificity. ${ }^{5}$ Usual clinical criteria (fever, lung auscultation, necessity of larger oxygen supply and/ or ventilation and purulent discharge from the tracheal tube) and usual laboratory criteria (two chest x-rays with new or progressive or persistent pulmonary infiltrate and blood test indicating infection) are not so specific and are relatively subjective and, therefore, whether used in isolation or combined, they can lead to misdiagnosis. Blood culture and pleural fluid culture, although they have good specificity, are poorly sensitive. ${ }^{1}$

Qualitative culture of tracheal aspirates, albeit regarded as sensitive $(90 \%)$, has low specificity $(40 \%) .{ }^{5}$ If collected improperly, the aspirate can be contaminated by airway bacteria; in addition, it cannot distinguish a simple colonization from a real infection. 2,5,7

Several studies (most of which involve adults, few including children, and almost none including newborns) seek to increase the specificity of the culture tests by using more sophisticated (and consequently more expensive) procedures in mechanically ventilated patients and in those suspected of having pneumonia. Although the quantitative culture $(\mathrm{CFU} / \mathrm{ml})$ and the presence of intracellular bacteria detected through the Gram staining of the collected material

\footnotetext{
* Full professor, Department of Pediatrics, Universidade Federal do Paraná (UFPR), Curitiba, PR, Brazil.
}

Suggested citation: Miyaki M. Sequential microbiological monitoring of tracheal aspirates in intubated patients admitted to a pediatric intensive care unit. J Pediatr (Rio J). 2005;81:3-4. improve the specificity for the diagnosis of VAP, they have not proved to be sensitive enough ( 30 to $78 \%$ ). ${ }^{1}$

Thus, the most current studies (included in the clinical practice of adult ICUs and in some pediatric ICUs as well) show that bronchoscopy with bronchoalveolar lavage (BAL), using a protected specimen brush (PSB), is the most appropriate methodology to collect the material, identify and quantify the pathogens. Flexible bronchoscopy for collection of the material leads to a large number of complications, and cannot be used in children with a thinner tracheal tube. On the other hand, the use of PSB is feasible in young children and has sensitivity and specificity similar to that of the BAL. $1,5,6$

In the literature, a consensus exists that it is better to use the available diagnostic resources (clinical examination, $\mathrm{x}$-ray, hemogram and cultures) together and not in isolation so that the best positive and negative predictive values can be obtained.

The study carried out by Carvalho et al. ${ }^{8}$, which consisted of the sequential bacteriological monitoring of tracheal aspirates in 100 pediatric patients submitted to MV, although it had some methodological limitations (material collected by an unprotected catheter, qualitative culture only, and nondetection of the presence or absence of intracellular bacteria in the aspirate material), is valid because it determined: 1 ) the bacteriological profile of the tracheal aspirate of mechanically ventilated pediatric patients in our setting; 2) rapid change of this bacteriological profile in a relatively short time of $M V ; 3$ ) the frequency at which tracheal aspirate cultures revealed the growth of more than one pathogen, thus interfering with the interpretation and validity of the results, especially with regard to the selection of the most appropriate antimicrobial to be used; 4) the predominance, after 96 hours, of Pseudomonas aeruginosa, the etiologic agent most frequently isolated in VAP; and 5) the frequency at which patients are colonized or infected by resistant bacteria (S. aureus MRSA and Klebsiella ESBL) and other pathogens (Candida) in our setting, which is a cause for concern among intensivists and infectologists.

However, some questions still have to be answered, such as the following: is tracheal aspirate culture useful in the diagnosis of VAP and in the selection of the most 
appropriate antimicrobial? Unfortunately, the available results are not enough to clarify this question. Only 10 out of 16 patients diagnosed with VAP had a positive tracheal aspirate culture and, among these, four patients revealed growth of more than one pathogen. Further studies are necessary to compare different methods in the pediatric population before we can make scientifically based recommendations. Hopefully, such measures will be able to provide our pediatric patients with improved quality of care.

\section{References}

1. Elward AM. Pediatric ventilator-associated pneumonia. Pediatr Infect Dis J. 2003;22:443-6.

2. Elward AM, Warren DK, Fraser VJ. Ventilator-associated pneumonia in pediatric intensive care unit patients: risk factors and outcomes. Pediatrics. 2002;109:758-64.
3. Urrea M, Pons M, Serra M, Latorre C, Palomeque A. Prospective incidence study of nosocomial infections in a pediatric intensive care unit. Pediatr Infect Dis J. 2003:22:490-3.

4. Apisarnthanak A, Holzmann-Pazgal G, Hamvas A, Olsen MA, Frazer VJ. Ventilator-associated pneumonia in extremely preterm neonates in a neonatal intensive care unit: characteristics, risk factors, and outcome. Pediatrics. 2003;112:1283-9.

5. Gauvin F, Lacroix J, Guertin M-C, Proulx F, Farrel CA, Moghrab $A$, et al. Reproducibility of blind protected bronchoalveolar lavage in mechanically ventilated children. Am J Resp Crit Care Med. 2002;165:1618-23.

6. Gauvin F, Dassa C, Chaibou M, Proulx F, Farrel CA, Lacroix J. Ventilator-associated pneumonia in intubated children: comparison of different diagnostic methods. Pediatr Crit Care Med. 2003;4:437-43.

7. Bush A. Bronchoscopy in pediatric intensive care. Pediatr Resp Rev. 2003;4:67-73.

8. Carvalho CE, Berezin EM, Pistelli IP, Mímica L, Cardoso MR. Monitoramento microbiológico seqüencial da secreção traqueal de pacientes intubados internados em UTI Pediátrica. J Pediatr (Rio J). 2004;81:29-33. 